\title{
Rapper en « terrain miné ». Pratiques musicales et dynamismes des imaginaires sorciers au Gabon
}

\section{Alice Aterianus-Owanga}

\section{(2) OpenEdition \\ 12 Journals}

Édition électronique

URL : http://journals.openedition.org/pa/93

DOI : $10.4000 /$ pa.93

ISSN : 2273-0362

Éditeur

Université Lumière Lyon 2

\section{Édition imprimée}

Date de publication : 31 octobre 2012

Pagination : 26-49

ISBN : 1634-7706

ISSN : 1634-7706

Référence électronique

Alice Aterianus-Owanga, «Rapper en «terrain miné ». Pratiques musicales et dynamismes des imaginaires sorciers au Gabon », Parcours anthropologiques [En ligne], 8 | 2012, mis en ligne le 20 avril 2013, consulté le 21 avril 2019. URL : http://journals.openedition.org/pa/93 ; DOI : 10.4000/pa.93 


\title{
Rapper en « terrain miné ». Pratiques musicales et dynamismes des imaginaires sorciers au Gabon
}

\author{
Alice Aterianus-Owanga
}

Université Lumière Lyon 2, CREA

Depuis ses prémices jusqu'à nos jours, l'histoire du mouvement rap au Gabon contient de nombreux récits, rumeurs et légendes urbaines faisant état de pratiques de sorcellerie dans les activités des rappeurs. Au milieu des années 1990, la mort prématurée d'un des précurseurs du rap à Libreville et les crises de folie qui la précédèrent furent ainsi associées à l'achat d'une bague «mystique » importée depuis l'Inde et employée comme un " fétiche » censé accroître le potentiel de réussite de son possesseur. Plus tard, un célèbre groupe gabonais, survivant à l'effondrement d'une rampe lumineuse lors d'un spectacle, se déclara dans un morceau rescapé des «attaques mystiques" envoyées par ses détracteurs et concurrents dans le réseau du rap, exprimant ainsi l'existence de sentiments de persécutions sorcellaires et de conflits mystiques à l'intérieur du milieu musical. Aujourd'hui encore, il n'est pas rare de voir dans les propos des auditeurs de rap ou des artistes des comparaisons entre certains rappeurs et des «ngangas », des "gourous », ou des féticheurs réalisant des «tchangs » pour parvenir au succès escompté ${ }^{1}$.

Ces différents récits illustrent la manière dont le mouvement rap est partie prenante d'un système de représentations symboliques et de relations sociales marqué par l'imaginaire sorcier. Le champ que je qualifie ici de "mystique » se manifeste en effet dans plusieurs sphères de la vie urbaine et de l'espace public gabonais ${ }^{2}$, comme le décrivent les travaux de Joseph Tonda (2005), Ludovic Mba Ndzeng (2006), ou Florence Bernault (2005), qui présente les soupçons sorciers comme le miroir reflétant toutes les tensions et conflits sociaux, et la sorcellerie comme " un enjeu crucial de l'imagination publique » (Bernault, 2005 : 21). A partir de données ethnographiques recueillies dans le cadre de plusieurs enquêtes ethnographiques auprès des rappeurs de

${ }^{1}$ Le nganga désigne le devin guérisseur dans différents rites initiatiques gabonais, notamment dans le bwiti. Le tchang est un objet confectionné lors d'un rituel visant à conférer une qualité ou un pouvoir particulier à son détenteur.

${ }^{2}$ La notion de « mystique » discutée au long de cet article provient des discussions populaires où elle qualifie des actions, des lieux, et des situations appartenant au registre de la sorcellerie, du spirituel ou de l'initiation. 
Libreville $^{3}$, cet article se propose de décrire comment le rap s'est implanté au sein de cet univers symbolique, et d'interroger les dynamiques sociales et les interpénétrations issues de cette rencontre. Tout d'abord, comment la pratique $\mathrm{du}$ rap s'est-elle intégrée dans des logiques d'interprétation et de représentations symboliques propres à ce contexte ? Par ailleurs, en quoi l'appropriation de cette forme musicale globalisée a-t-elle contribué, de manière réciproque, à l'émergence de nouvelles dynamiques de l'imaginaire sorcier?

Face à l'épineuse question de la «réalité » de la sorcellerie et des préceptes méthodologiques et épistémologiques à adopter pour aborder des terrains discutant des phénomènes surnaturels, la notion d' " imaginaire sorcier » (ou $d^{\prime}$ « imaginaire de la sorcellerie ») traverse les écrits de différents chercheurs en sciences humaines, s'y présentant comme un concept analytique opératoire ${ }^{4}$ (entre autres : Dozon, 1981 ; Bernault \& Tonda, 2000 ; Geschiere, 2000 ; Mary, 2001). Dans la continuité de ceux-ci, je m'appuie sur le concept d'imaginaire sorcier pour désigner un cadre de déchiffrement du réel se référant à des lieux, des actions ou des instances appartenant au registre du surnaturel. J'emploierai donc dans cet article l'expression émique de "mystique » retrouvée à plusieurs reprises dans les expressions des jeunes Gabonais, ainsi que celle $\mathrm{d}^{\text {' } ~}$ imaginaire sorcier », qui relève d'une catégorie d'analyse étique des discours et phénomènes observés.

Dans un premier temps, je décrirai globalement comment les textes des rappeurs véhiculent des lignes structurantes de l'imaginaire sorcier et en quoi la pratique du rap s'est intégrée dans ce terrain "miné » ${ }^{5}$ par la sorcellerie. Puis, j'analyserai les morceaux de rap d'un rappeur de l'ethnie $f a n g^{6}$ évoluant entre Libreville et l'intérieur du Gabon et leurs fonctions dans son vécu social, familial et religieux, pour mettre en évidence la manière dont la pratique musicale contribue aux métamorphoses de l'univers sorcier et de ses logiques sociales, cela en résonance avec les influences de l'expérience religieuse initiatique. Je m'intéresserai enfin à la manière dont le rap est employé comme instrument de liaison ou de redéfinition des frontières entre différentes catégories de la vie sociale et symbolique : entre la province et la capitale, entre les aînés et les cadets sociaux, entre l'univers du jour (et des humains) et

${ }^{3}$ Ces enquêtes réalisées entre 2007 et 2011 s'inscrivent dans le cadre d'une thèse de doctorat en anthropologie s'intéressant aux pratiques musicales en contexte de mondialisation et aux processus identitaires développés autour de l'implantation du rap au Gabon.

${ }^{4}$ Peter Geschiere note ainsi dans ses réflexions sur les relations entre sorcellerie et modernité que " l'imaginaire sorcier imprègne et conditionne la manière dont les gens essaient d'interpeller les changements modernes » (Geschiere, 2000 : 26).

${ }^{5}$ Je reprends ici les paroles d'un morceau (à paraître) du rappeur présenté dans cet article, Roda N'No.

${ }^{6}$ Les Fang sont l'ethnie démographiquement majoritaire du Gabon, localisés dans la province du Woleu-Ntem (Nord du Gabon), dans l'Estuaire et d'autres provinces du Centre du Gabon. Le mot Fang désigne aussi leur langue. 
celui de la nuit (des sorciers). Il convient cependant d'abord d'entamer cette contribution par quelques éléments de contextualisation concernant l'implantation du rap dans la société gabonaise et l'entrée de la sorcellerie dans le milieu social des rappeurs.

\section{L'ENTRÉE DE LA SORCELLERIE DANS LES DISCOURS ET LES PRATIQUES DES RAPPEURS}

Précédé par la création de groupes de danse et la reprise d'un ensemble d'attitudes corporelles inspirées des États-Unis, le rap apparaît à Libreville à la fin des années 1980, dans le contexte de crise politique et de revendication démocratique précédant l'instauration du multipartisme (1991). Alors que les concours de breakdance et de "robotique " font déjà fureur depuis plusieurs années à Libreville, V2A4 est le premier groupe de rap gabonais à se faire entendre dans les écrans et les postes radiophoniques librevillois en 1990, avec le morceau "African Revolution». Ces enfants de hauts fonctionnaires gabonais y signent une diatribe dénonçant la corruption, le monopartisme et la conservation du pouvoir entre les mains des élites. Le mouvement rap se propage alors rapidement auprès des jeunes de milieux plus défavorisés, pour se développer au cours des années 1990 par la formation de labels de production musicale, l'organisation en réseau des acteurs de la musique rap ${ }^{8}$, et leur mise en relation avec le marché local et international de la musique. Aujourd'hui, les studios d'enregistrement et les médias de diffusion de la musique rap se localisent principalement à Libreville, où réside la quasiintégralité des artistes en avant de cette scène musicale?. Certains ont grandi dans des villes ou des villages de l'hinterland gabonais, d'autres, du fait des mobilités interprovinciales qui traversent le territoire gabonais (entre autres en raison des mutations des fonctionnaires et agents administratifs), ont parfois résidé dans une ou plusieurs villes de province.

Après s'être focalisés sur la dénonciation des modes de gouvernance autocratiques, du système néocolonial (marqué par la présence des militaires français à Libreville), ou des conditions de vie précaires des Gabonais, les

${ }^{7}$ La robotique était dans les années 1980 l'une des danses diffusées par le biais de la vague hip-hop.

${ }^{8}$ Je qualifie de réseau du rap un ensemble d'acteurs participant de la production musicale rap au Gabon (rappeurs, producteurs, managers, journalistes, concepteurs musicaux, organisateurs de spectacles...), liés par des relations de densités et de natures diverses, et réunis au sein d'un emboitement de formations sociales. Je m'inscris ainsi dans la continuité de certains courants d'anthropologie urbaine (Hannerz, 1983), et des recherches récentes qui ont mis en avant l'intérêt de considérer les pratiques artistiques et musicales au prisme de ce cadre d'analyse (Hammou, 2010), notamment en écho avec les processus de transnationalisation (Argyriadis, 2009).

9 On notera que la capitale économique Port-Gentil regroupe également un petit nombre d'artistes fonctionnant relativement en autonomie de Libreville. 
textes de rap se sont progressivement intéressés à différents autres aspects de la vie des jeunes de Libreville: les relations de genre, les ambiances festives nocturnes, la vie urbaine, le milieu du rap et son organisation, mais aussi la thématique de la sorcellerie ${ }^{10}$. Le fétichisme et les pratiques occultes (trafic d'organes, consommation de sang humain, sacrifices, crimes rituels) sont partie intégrante du quotidien urbain, des faits divers et des imaginaires populaires, ce pourquoi la question de la sorcellerie constitue une thématique à part entière des textes de certains rappeurs. Outre des allusions fréquentes aux notions de "vampire», de "sorcellerie» et de "magie », on trouve quelques morceaux consacrés exclusivement à la question du mystique : le titre "Blindé », référence aux techniques et rituels de protection visant à entourer l'individu d'un «blindage », "Me vine mbo », qui en langue fang signifie « je n'aime pas la sorcellerie », ou encore "Ékowong » ${ }^{11}$, nom d'un village situé dans une province du nord-est du Gabon connue pour ses nombreux sorciers.

Les pistes consacrées à la question du « mystique » et des pratiques rituelles sont fréquemment combinées à des accompagnements instrumentaux traditionnels et parfois à des textes en langue vernaculaire. Loin d'être anodine, cette observation sur les liens entre l'alternance linguistique et thématique dans le rap révèle des logiques structurantes de l'imaginaire sorcier. D'après les explications recueillies auprès de rappeurs et rappeuses abordant ce sujet, le domaine de la sorcellerie comporte un trait commun avec les langues vernaculaires, les instruments traditionnels et les pratiques initiatiques : leur origine villageoise. Considérées comme propres aux cultures gabonaises et antérieures aux phénomènes $\mathrm{d}$ 'acculturation nés de la rencontre avec l'Occident, certaines thématiques sont abordées dans les textes de rap en langue vernaculaire, car elles comporteraient le même rattachement à l'univers villageois, berceau originel d'un ensemble de pratiques depuis lors en mutation dans le contexte urbain. Un rappeur de Libreville m'expliquait ainsi à ce sujet : "C'est un cliché, mais pour nous le spirit, le vampire, c'est le bled. [...] Si les gens fuient le bled, l'exode rural, c'est à cause du vampire » (BJL, août 2011, Libreville). Les zones provinciales, ici désignées au travers du nom argotique «bled » sont dans les stéréotypes populaires des jeunes de Libreville en corrélation avec les pratiques de sorcellerie comme « le vampire » ou son synonyme argotique, le «spirit».

En outre de son imprégnation dans les textes des rappeurs, l'omniprésence de la sorcellerie se donne également à voir dans des modes de relation entre

10 Les travaux de sociolinguistique réalisés par Michelle Auzanneau (2001) apportent un aperçu des thématiques abordées dans les textes de rap gabonais.

11 Le titre «Blindé » est extrait de l'album «Bienvenue à LBV », Lestat XXL, Zorbam Produxions, 2007. Le morceau «Me vine mbo », du rappeur Harley B., date du début des années 1990 et n'a été conservé ni dans des archives privées ni dans des banques de données radiophoniques. Le titre « Ekowong » est enfin issu du double album en cours de parution du rappeur Roda N’No (2012), « Akamayong / Nkemeyong ». 
les rappeurs et les groupes de rap, qui témoignent de stratégies d'accroissement de la notoriété et d'une conflictualité employant, entre autres, le canal d'armes «mystiques». Alors que dans le milieu de la rumba congolaise de Kinshasa, Bob White observe que les rumeurs de sorcellerie touchent particulièrement les artistes musiciens qui « $\mathrm{s}^{\prime}$ affirment de manière indépendante des institutions sociales et des réseaux $»^{12}$ (White, $2008: 230$ ), le milieu musical gabonais est aussi parcouru en différents points par des rumeurs de pratiques occultes. Traitant d'opérations mystiques, d'usages de fétiches, de bains purificateurs, de rituels occultes, et d'attaques entre artistes au travers de «fusils nocturnes" ou de «CPT»" ${ }^{13}$, les comportements, les rumeurs, et les discours recueillis auprès des artistes permettent de penser que la sorcellerie a pleinement pénétré cet univers artistique pour devenir une arme stratégique de la concurrence entre artistes. Ils confirment en ce sens l'idée d'une entrée de la sorcellerie dans de nouvelles sphères de la vie urbaine $^{14}$, et son extension en dehors de la sphère intralignagère longtemps étudiée par les anthropologues africanistes (Adler, 2006) - bien que cette dernière conserve une place importante dans l'imaginaire et le vécu des rappeurs observés. Nous allons voir au travers du cas d'un artiste qu'en parallèle d'une pluralisation des formes du mystique dans le milieu des musiques urbaines, la rencontre et l'interpénétration entre rap et «territoires sorciers » (Henry, Tall, 2008) conduisent à une redéfinition de certains thèmes majeurs de l'imaginaire sorcier que sont la sphère familiale, le village et les ainés sociaux.

\section{RAP DE «PROVINCE » ET DÉSIRS D’ENTRÉE DANS LE MARCHÉ INTERNATIONAL DE LA MUSIQUE}

Auteur de certains des morceaux de rap gabonais les plus riches en matière

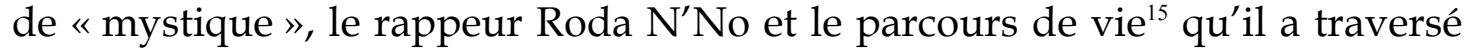
apportent des angles d'entrée tout particulièrement intéressants pour

12 Je traduis les propos de Bob White (2008), qui met en exergue les relations entre les rumeurs desorcellerie et les sanctions communautaires contre les attitudes individualistes. Pour un article consacré à la question des rumeurs de sorcellerie chez les musiciens de Kinshasa, voir aussi White (2007).

${ }_{13}$ Ce concept est inspiré d'un service offert par les opérateurs de téléphonie mobile, qui permet le transfert de crédit d'un abonné à un autre. Dans les discours populaires sur la sorcellerie, il s'agit d'une attaque ou d'une affection mystique se projetant par l'intermédiaire du téléphone. Un morceau du groupe de rap 241 intitulé «CPT» (2008) fait par exemple le récit des attaques mystiques dont ces artistes seraient victimes.

${ }^{14}$ Pour quelques ouvrages traitant des dynamiques urbaines et évolutions contemporaines de la sorcellerie, voir par exemple Moore \& Sanders (2001), Meyer \& Pels (2003), ou encore le numéro « Territoires sorciers » des Cahiers d'études africaines (Henry \& Tall, 2008).

${ }^{15}$ Les informations concernant les œuvres et le récit de vie de Roda N'No sont publiées avec l'accord de cet artiste. 
appréhender les interactions entre l'activité musicale et les pratiques ou les représentations liées à l'imaginaire sorcier.

De par les liens singuliers qu'il entretient à l'égard des zones provinciales et de l'univers du "mystique», ce dernier offre tout d'abord un axe de compréhension original des relations entre la pratique du rap, les circulations entre rural et urbain, et la question de la sorcellerie. A la différence de ses pairs, qui sont issus d'itinéraires de vie en contexte urbain, Roda N'No a été élevé durant la majeure partie de son enfance et de son adolescence dans la province de l'Ogooué-Ivindo (nord-est du Gabon), et il se définit comme un enfant de "mkwakh", désignation spatiale attribuée aux zones situées en périphérie urbaine, aux confins des routes bitumées et à l'orée des zones de brousse. Ces espaces sont souvent associés dans l'imaginaire des gens de la ville à un degré de « développement » ou de "civilisation » inférieur, et à la condition de "broussard», pour reprendre le terme péjoratif employé par nombre de librevillois à l'encontre des personnes du village ${ }^{16}$. Cette dépréciation de certains espaces ruraux et des origines territoriales "villageoises » fait écho aux associations d'idées remarquées précédemment entre le village et des pratiques perçues comme rétrogrades, telles que la sorcellerie.

C'est depuis Makokou - chef-lieu de la province de l'Ogooué-Ivindo - que Roda N'no découvre au début des années 1990 quelques figures du rap français comme Iam, qui le marquent par la profondeur et la portée lyrique de leurs textes. Tout en s'intéressant à la culture hip-hop en pleine expansion à Libreville et à l'internationale, mais encore embryonnaire dans la ville de Makokou et sa périphérie, il poursuit son parcours scolaire et son quotidien de jeune du village, ponctué par les activités rurales et par les étapes d'initiation aux rites traditionnels, comme celui qu'il traverse à six ans à l'occasion de sa circoncision. Tandis qu'il écrit ses premiers textes à la fin des années 1990, des groupes de rappeurs se constituent dans les quartiers et établissements scolaires de Makokou, réunis par des liens ethniques ou lignagers. En concordance avec la composition ethnique de cette ville, les collectifs scindent alors les jeunes de l'ethnie fang et ceux des groupes Kwélé et Kota, les principales ethnies qui se répartissent dans les quartiers de la localité. Suite à une période d'affrontements entre ces clans de rappeurs fondés sur un ciment ethnique et lignager, plusieurs de leurs membres se réunissent au sein d'un groupe, "6 6 ième Cime », qui comme son nom l'indique, envisage de mettre en avant les richesses de la 6 ième province du Gabon (l'Ogooué-Ivindo) dont ils se reconnaissent.

16 Un rappeur originaire d'une ville du sud du Gabon m'expliquait ainsi le stéréotype entretenu par une part de la population de Libreville au sujet du «villageois » : "On a l'impression que c'est un abruti. On a l'impression qu'un villageois, c'est quelqu'un qui ne connait rien, qui ne défend rien, qui connait peut-être juste la chasse, planter, manger, dormir. Je pourrais même aller plus loin: on a même l'impression qu'un villageois, c'est quelqu'un qui n'est jamais allé à l'école » (Buung Pintz, 8/08/2011). 
éAu début des années 2000, ce groupe se stabilise autour de trois membres principaux : Roda N'No, de l'ethnie fang, D.Golss, rappeur kwélé d'un village voisin de Makokou, et Bubal, chanteur punu originaire du sud du Gabon. Ils profitent de vacances à Libreville pour enregistrer leurs œuvres musicales dans des studios (alors inexistants dans la majeure partie des villes de province), et les ramener ensuite à Makokou.

A la différence du Liban, où Nicolas Puig (2009) observe que le rap "témoigne de l'urbanité des individus et constitue une matrice de représentations des quartiers et des camps » (Puig, 2009: 166), nous voyons ici que la territorialité de ces acteurs du rap gabonais se définit dans un dialogue et une circulation entre pôles urbains et ruraux. Quoiqu'ils se revendiquent en partie d'une culture hip-hop urbaine, c'est en affirmant leur éducation traditionnelle du «bled » et en mettant en avant dans leurs morceaux et leurs comportements les savoirs acquis au travers des initiations traditionnelles et du quotidien des enfants du village qu'ils s'octroient la singularité artistique recherchée vis-à-vis des autres rappeurs de Libreville. Ainsi, tandis que Roda $\mathrm{N}^{\prime}$ No expose dans ses morceaux des techniques de chasse, systèmes de plantation agricole ou habitudes culinaires du village sous la forme de proverbes, de figures de style et de métaphores, D.Golss s'attache de son côté dans certaines œuvres en langue kwélé à retracer le récit de sa circoncision, rite de passage fondamental de la vie sociale et symbolique des Kwélél ${ }^{17}$.

C'est d'abord au sein du groupe 6ième Cime que Roda N'No évolue musicalement, puis lors de son installation à Libreville, où il passe son baccalauréat et s'inscrit à l'université, il entame une carrière en solo. A partir de 2007, il se fait connaitre dans le réseau du rap de Libreville par ses textes en langue fang nzaman et par les fonds instrumentaux traditionnels qui accompagnent ses morceaux, se faisant produire durant quelques années par le label ${ }^{18} \mathrm{~d}^{\prime}$ un groupe de rap évoluant entre la France et le Gabon (Zorbam Produxions).

Après avoir exercé auprès de ce label, puis suite à des rencontres avec des musiciens étrangers lors d'un festival se tenant à Libreville ${ }^{19}$, sa démarche musicale actuelle s'oriente autour de la recherche d'une authenticité musicale. Celle-ci résiderait dans la mise en avant des langues vernaculaires et de

${ }^{17}$ Chez les Kwélé, la circoncision des jeunes garçons marque leur passage à l'âge d'homme par un ensemble d'épreuves et festivités, organisées annuellement pendant la période de saison sèche (qui s'étend de juin à septembre).

$18 \mathrm{Au}$ Gabon, le terme label désigne dans la majeure partie des cas des organisations informelles d'entraide et de production musicale, et dans quelques rares cas, des structures officielles établissant des contrats.

${ }^{19}$ Le festival Gabao qui se tient annuellement entre la fin du mois de juin et le début du mois de juillet constitue depuis 2001 une des principales plateformes de mise en relation entre les rappeurs de Libreville et l'international, puisqu'il reçoit des artistes et entrepreneurs culturels étrangers (directeurs de festivals, producteurs, agents de maisons de disque). Roda N'No travaille ainsi depuis 2008 avec un concepteur musical résidant en France, rencontré à l'occasion de sa performance lors de cette édition du Gabao. 
connaissances locales traditionnelles acquises auprès des anciens du village ${ }^{20}$. En réponse à la quête de l'authentique décrite par certains chercheurs comme un trait du marché postcolonial de la musique ${ }^{21}$ (Raibaud, 2009; Da Lage, 2000, 2009), Roda N'No envisage d'aller puiser dans les «profondeurs » de la langue fang, pour la réinventer au son des rythmiques hip-hop et des arrangements de concepteurs musicaux basés en France et en Angleterre, et ainsi se faire connaitre par-delà les frontières du Gabon dans les réseaux de la "world music». Ce désir de fabrique d'un style musical original, commercialisable dans les marchés musicaux nationaux et internationaux, le conduit à rechercher des éléments de particularisation typiquement africains ou fang dans les traditions, les langues ou les pratiques religieuses locales, ce qui explique en partie la place particulière accordée à la sorcellerie et au « mystique » dans ses morceaux. Roda N'No introduit en effet dans son œuvre de nombreux récits de sorcellerie et des références à l'univers symbolique, qu'il s'agisse des rituels d'initiation traditionnels qu'il a traversés ou des soupçons de maléfices qui auraient été jetés dans sa parenté.

\section{UNE ESTHÉTIQUE ÉGOTRIP ENTRE CONDAMNATION SORCIÈRE ET RÉCIT INITIATIQUE}

L'inclusion du champ du mystique dans la pratique du rap donne naissance chez Roda N'No à une version originale de la notion d'égotrip. Ce type de discours, exercice de style compétitif jouant sur des techniques de vantardise, se retrouve dans plusieurs adaptations du genre musical rap au travers du monde (Barret, 2008 ; Moulard-Kouka, 2008 ; Pecqueux, 2009). Pour les rappeurs du Gabon, les textes intitulés " égotrip » peuvent être selon les cas des exercices de vantardise basés sur la démonstration de l'élégance vestimentaire, de la masculinité, du talent rhétorique ou de la force du rappeur, ils peuvent se fonder sur des récits autobiographiques ou des narrations de soi, ou encore procéder par des défis et provocations aux adversaires rappeurs en vue de démontrer la témérité de l'auteur. Mais dans l'écriture de Roda N'No, comme chez d'autres artistes appartenant aux sociétés initiatiques locales, l'égotrip s'associe fortement aux questions de sorcellerie et au récit du parcours initiatique. Les textes qu'il classe dans ce registre consistent en des mises en récit de soi combinant la projection imaginaire dans le monde du village, la présentation de la généalogie lignagère et l'univers symbolique de la sorcellerie. S'appuyant sur l'exercice

${ }^{20}$ Les morceaux de Roda N'No et l'usage du fang qu'il y fait sont le produit de recherches effectuées auprès des anciens des villages Mayiga et Ekowong, respectivement à sept et cinq kilomètres de Makokou.

${ }^{21}$ Ces canons du marché de la world music sont relayés auprès des artistes de musique urbaine de Libreville par le biais de radios comme RFI, des institutions culturelles françaises locales ou de festivals internationaux de musique, tels que le festival Gabao cité plus haut. 
de célébration de soi et de vantardise caractéristique du genre rap, l'œuvre de Roda N'No met alors en place un dialogue entre la dénonciation de la sorcellerie et la référence au monde de l'initiation, deux univers interdépendants.

La relation entre initiation et condamnation sorcière est probante dans l'un des morceaux de Roda N’No nommé « Mayi » (en langue fang, «je pleure ») ; il y pleure la perte de sa mère et de ses parents décimés par l'action des sorciers, en citant les noms des membres de sa famille responsables de leur mort. Il condamne les pratiques de sorcellerie et le détournement des techniques traditionnelles d'accumulation de force (comme le byéri ${ }^{22}$ ) au profit d'actions maléfiques, en interpellant ainsi ses défunts parents :

«A N'no Missoune, Evoue Missoune,

A Nze Missoune.

Mi nga wu e dzè ?

DzôgOa me ye ngwel ye

mam me ya nsône?

Ye mia yem na bi mina ke be mi ngengang

ka Dzeng dzôm é nga mine yü ?

Évine nyo, asông nze, ôvône beyem ?

BiyogCe B'etome, NzogCe, Nze,

bî mina be yem.

Roda N'No a ne ndzi ébôgOa

a sira fe ndzem.

Bong bese mi nga likOnda be mina vem nom de ses oncles maternels

nom de sa mère

Qu'est-ce qui vous a tués?

Dites-moi, est-ce de la sorcellerie

ou bien une mort naturelle?

Savez-vous qu'on est allés jusqu'à consulter

des ngangas pour connaître l'origine de votre mort?

Est-ce le mamba noir, la dent de la pantCère, la OacOe des sorciers ${ }^{23}$ ?

BiyogCe B'etome, NzogCe, Nze [grandsparents de Roda],

on les connaît.

Roda N'No a déjà mangé le bois sacré

il n'est plus dupe.

Tous les enfants que vous avez laissés ont grandi

${ }^{22}$ Le byéri est un culte des ancêtres de l'ethnie fang, fondé sur la conservation des ossements et des crânes ancestraux dans des boites reliquaires dont la transmission est censée assurer la prospérité du lignage.

${ }^{23}$ Ces trois éléments sont des objets utilisés au titre de fétiches, ou comme des reliques léguées par les ancêtres, détentrices d'un pouvoir mystique. Le mamba noir sert à protéger la famille, et assure sa prospérité en renfermant la puissance du chef de famille. Ces pouvoirs sont aujourd'hui considérés comme maléfiques, et objets de crainte, associés aux rites occultes et aux pratiques de sorcellerie. 
Ekila, Missoune, Missoune,

Emane, N'no, Ngningone, Nyingone.

Be te be fam, bôra be ngone.

Be te bo bibone.

Ma yagOa na be ne ke ke melukO

ke byè bone.
Ekila, Missoune, Missoune,

Emane, N'no, Ngningone, Nyingone. ${ }^{24}$

Ils sont devenus des Oommes et des femmes

Ils sont en âge de se marier.

C'est bizarre que les femmes ne trouvent pas d'époux

et n'enfantent pas... ${ }^{25}$

Dans ce premier couplet, Roda N'No révèle l'identité des responsables de l'infortune qui touche sa famille (stérilité, célibat, morts inexpliquées), contenue en la personne de certains de ses grands-parents et aînés du village. Il nomme les auteurs des meurtres de ses parents, en justifiant ses dires à partir de l'expérience initiatique qu'il a traversée et des découvertes qu'il a faites en ingérant le "bois sacré ». Cette expression désigne dans le langage populaire la plante locale tabernanthe iboga, dont la consommation est au cour de différents rites initiatiques gabonais. De même lorsque Roda N’No déclare ne plus être «dupe », il sous-entend qu'il a été initié, et qu'il a par ce canal pris connaissance des ennemis à l'origine de son infortune.

Le premier rite de passage que Roda $\mathrm{N}^{\prime} \mathrm{No}$ a traversé à sa naissance est celui du melan, la plus grande société secrète des Fang. Ce rite se fonde sur le culte des ancêtres, opéré par l'intermédiaire du byéri, reliques sacrées composées de crânes et d'ossements, auxquelles est présenté l'initié pour le protéger et l'instruire des secrets du lignage. Ce n'est cependant pas à cette initiation que Roda N'No fait référence dans son morceau, mais à celle qu'il a vécue beaucoup plus tardivement : l'initiation au mbiri.

Le mbiri des Fang est une variante ou une branche du bwiti, fondé sur la consommation de l'hallucinogène iboga afin de provoquer un voyage visionnaire $^{26}$. Roda N'No s'y est initié en 2006, à Libreville, à l'initiative de son frère et de sa sœur ainés. Alors qu'il fréquentait déjà les veillées de bwiti de manière profane ${ }^{27}$, il fut appelé par ces derniers, désireux de franchir l'étape

24 Il s'agit des enfants de la famille de Roda N’No demeurés orphelins.

${ }^{25}$ Les extraits cités dans cet article ont été traduits en français en collaboration avec leur auteur. La transcription orthographique s'est effectuée en partie avec l'aide du dictionnaire de Samuel Galley (1964), mais elle s'est surtout appuyée sur les règles de l'alphabet scientifique des langues du Gabon (Hombert, 1990). Nous avons choisi de ne pas spécifier la tonalité ou la longueur vocalique des termes, en partie car ces éléments sont d'un intérêt secondaire pour une étude d'anthropologie, et car la prononciation du Fang nzaman employé par Roda N'No diffère sous plusieurs aspects des autres variantes de cette langue.

26 Pour les bwitistes, le mbiri est parfois appelé « l'hôpital » car il comporte une finalité spécifiquement thérapeutique (Fernandez, 1982 : 595).

${ }^{27}$ Les veillées et les temples de bwiti séparent en effet les lieux et les moments accessibles aux profanes non-initiés, de ceux réservés aux seuls initiés. La dichotomie profane / initié est un 
initiatique pour résoudre des sentiments de blocage dans leurs vies professionnelles et des maladies chroniques. Après s'être munis des objets requis pour l'initiation (nattes, pagnes, seaux, bougies), ils traversèrent d'abord tous trois une période de préparation passant par des bains purificateurs, et par le respect de certains interdits alimentaires et sexuels. Puis ils accomplirent le cœur de l'initiation dans le temple de la nganga (mèreinitiatrice): enfermés durant plusieurs jours dans une chambre, ils consommèrent la poudre de racine d'iboga, effectuèrent leur voyage visionnaire et découvrirent les causes de leur infortune, ou les sorciers à l'origine de leurs malheurs. Porte d'accès au monde "mystique", cette initiation fut le moyen pour Roda N'No d'entrer en contact avec ses ancêtres et son esprit tutélaire.

$C^{\prime}$ est au retour de cette initiation, après quatre jours passés dans le temple de la guérisseuse, que Roda N'No vît en songe sa mère décédée, et qu'il écrivit le texte «Mayi ». Assis sous le manguier situé au front de sa demeure familiale de Libreville, il créa ce morceau en un jour, inspiré par l'état ressenti durant la phase post-initiatique et par les effets hallucinogènes de l'iboga. Il conçut son œuvre en l'absence de musique, en commençant par ce couplet où il adopte la voix d'un esprit qui s'adresserait ainsi à lui :

« A mone venengOe, ke fangOa yi !

nyüe aligOa we émo,

ô ne mam fam,

tangOi lumengCe fimi mir !

Ô bele nlô, ô bele mir, ô bele züi,

ô bele anyu, ô bele mo,

yemi bè, a tare, ô sira ndzem !

abyali dia, be ve w'éyôle ;

N'no Missoune, ndôm nyüe

TagObe ane fam !

K'akône nda, mi ligAi ya nseng

étam étam. Nzame a ta mine.

A Nkorebore volegCe bone,

bôr be si nyeng e kegCe ôwoni, énam ku beloma ${ }^{28}$, ntsima ékone, éfu akwagOa ba mendzim meyông ${ }^{29}$.
Mon enfant, sois fort, ne pleure plus ! ta mère t'a donné la vie sur terre.

$\mathrm{Tu}$ es un garçon, calme-toi et ressaisis-toi!

Ta mère t'a doté d'une tête, d'yeux, d'un nez, d'une boucOe, de mains.

Ecoute bien, tu es un garçon sensé !

A ta naissance, on t'a donné un nom ;

N'no Missoune, celui de ton oncle maternel,

Comporte-toi comme un Oomme !

Même si vous n'avez pas de protecteur et que vous êtes restés seuls, Dieu vous regarde.

Nkorebore, aide tes enfants, parce qu'on

ne peut vivre que de graines d'aracOide, de repas de misère, et

des décoctions que nous donnent les anciens ».

élément structurant de cette société secrète (Bonhomme, 2006), de même que dans d'autres cérémonies initiatiques (Jaulin, 1967).

${ }^{28}$ Dans son canton, cette expression qui signifie littéralement « les ailes de poule aux feuilles de taro » représente le déjeuner des enfants : du manioc avec de l'huile, du piment et du sel.

29 "Meyong » désigne une décoction aux fonctions médicinales préparée par les anciens à partir de graines et d'herbes de brousse, et bue le matin accompagnée de tubercules de manioc. 
Le nom «Nkorebore» auquel fait référence l'esprit s'exprimant dans ce texte n'est autre que le kombo de Roda N'No; rapporté de son voyage initiatique, ce nom constitue l'un des marqueurs de la mort symbolique et de la renaissance de l'individu survenant lors de l'initiation (Mary, 1983). Dans le bwiti, le kombo est toujours supposé être en adéquation avec la personnalité de son porteur et chargé d'une signification ésotérique; dans le cas de Roda N'No, Nkorebore désigne un sauveur, venu délivrer son peuple. Cette fonction rédemptrice découverte par Roda N'No est invoquée dans ce texte comme clé de résolution des maux familiaux, à l'origine d'un comportement nouveau contre les attaques sorcières et les malédictions qui frappent sa famille. L'initiation est donc décrite comme l'embrayeur d'une réaction contre la sorcellerie et d'une prise en main du vécu lignager, confirmant les analyses de Julien Bonhomme sur le rôle de l'initiation au bwiti pour une reconfiguration des relations entretenues par l'individu avec le lignage et les sorciers (Bonhomme, 2005a).

Parallèlement, ce morceau de Roda N'No intéresse notre présente discussion en ce qu'il met en évidence l'existence d'autres reconfigurations conséquentes à l'initiation, agissant cette fois dans le domaine de la création musicale. Devenant un élément moteur de la création rap, l'initiation au bwiti (ou à sa variante mbiri) et la reconfiguration du vécu qu'elle entraîne modifient les formes de la création et les contenus des œuvres musicales. On notera tout d'abord que ce morceau a été écrit dans un état mental fortement nourri des effets hallucinogènes de l'expérience initiatique, conférant une signification singulière à la notion d'inspiration.

De plus, les expériences initiatiques sont réintégrées selon l'organisation du discours et de l'attitude affichée dans le genre rap : son kombo, nom d'initiation soumis usuellement à une part de secret, se voit exposé et brandi au même titre que les pseudonymes, «blaze» ou autres «a.k.a» déclinés dans les performances des rappeurs. Il sert, de même que la référence au statut d'initié, un propos centré sur l'affirmation du soi, qui joue sur la démonstration d'un pouvoir mystique conférant au rappeur une aura supplémentaire. Chez les rares rappeurs de la scène locale appartenant à des sociétés initiatiques, on retrouve fréquemment cette fusion des esthétiques égotrip et de l'affirmation du pouvoir spirituel ou des savoirs initiatiques qui conduisent dans la société urbaine gabonaise à un surcroit de considération, d'estime et de crainte. S'affirmer "blindé », chanter la grandeur de sa force spirituelle ou défier ses adversaires de l'agresser mystiquement constituent autant de modalités d'agrandissement de soi et d'autocélébration des rappeurs, et autant de témoignages de la pénétration du champ de la sorcellerie au sein des modes de conflictualité et de défi propres au genre rap.

Chez Roda N'No, les apports et modifications induits par l'initiation dans le vécu individuel, déclencheurs d'une nouvelle attitude vis-à-vis de la sorcellerie et du lignage, sont réinvestis selon les normes de présentation de cet univers musical, conduisant comme nous allons le voir dans la partie 
suivante à une remise en question de certains rapports de force et de domination. Car à la différence de l'égotrip de morceaux de rap classiques, Roda N'No ne s'adresse en effet pas seulement dans ses textes à des potentiels adversaires rappeurs, mais à ses sorciers et à certains membres de sa parenté.

\section{DE L'ACCUSATION SORCIÈRE AU PROCÈS DES AÎNES SOCIAUX}

Dans le corpus d'œuvres de Roda N'No, plusieurs textes traitant du «mystique" comportent des références combinées entre l'univers de l'initiation et celui de la sorcellerie, et se rejoignent par le portrait identique du sorcier qu'ils dépeignent, sous les traits du vieillard. Dans le texte "Ekowong", Roda N'No procède par exemple à une description des pratiques de sorcellerie ayant cours chez les anciens du village et il décrit les actions de ses grands-parents et aînés sociaux par les expressions suivantes : "réaliser leurs sombres projets», "planter leurs fétiches tout autour du terrain familial ", "miner les sentiers de pièges en tous genres", " extraire [s]on sang dans le but de nourrir leur animal totémique», ou encore "maudire ses enfants, tuer ses proches parents". Il désigne d'abord ses accusés au travers du pronom «ils", puis cite nommément un grand-père sous la désignation «ce satané Nzoghe Obame » à qui il reproche d'avoir jeté la malédiction sur sa famille. Légitimant ses accusations par un recours aux aïeux et à la "tradition $»^{30}$, il incrimine finalement ses grands-parents de la manière suivante: "sachez que nos parents chassaient pour nourrir leurs enfants, et non pour que ceux-ci soient consommés par leur serpent $»^{31}$.

Le morceau «Ekowong» rend compte des sentiments de persécution sorcellaire exprimés par certains jeunes Gabonais, et de l'association généralement effectuée entre la sorcellerie, le village, et les anciens, perçus comme les consommateurs des forces et de la vitalité de la jeunesse. Chez Roda N'No comme chez d'autres rappeurs, la représentation du vieillard sorcier et de la consommation des forces des jeunes enfants par les anciens du village est un trait récurrent, de même que la personnification du sorcier dans la figure du vieillard, que l'on retrouve couramment dans les discours sur la sorcellerie $^{32}$. Dans les textes de Roda N'No, si la vieillesse symbolise en un

30 Roda N'No établit en effet une distinction entre l'usage des forces occultes fait autrefois par ses aïeux, qui conservaient des reliques et utilisaient leurs savoirs en vue d'accroitre le capital familial, et l'usage qui en est fait aujourd'hui, aux finalités maléfiques.

${ }^{31}$ Le serpent, associé dans l'imaginaire populaire à la sorcellerie, était autrefois un animal employé dans les familles comme relique contenant la force du lignage. Comme Léa Zame Avezo'o le note dans une étude sur la néo-oralité urbaine, cette figure "s'enracine dans de nombreuses sociétés gabonaises qui associent le serpent, notamment le python, à l'abondance et à la régénération permanente » (Zame Avezo'o, $2005: 235)$.

32 Cette propriété est toutefois remise en question depuis quelques années par le phénomène des enfants sorciers (De Boeck, 2000 ; Yengo, 2008), que Filip De Boeck analyse comme «la 
sens la sagesse et la connaissance traditionnelle, elle est aussi l'origine des maux actuels de la jeune génération et la source des blocages mystiques qui l'astreignent à des conditions de vie précaires. Pour les jeunes du Gabon, bien que le respect du droit d'aînesse constitue un principe fondamental ${ }^{33}$, les anciens du village sont les principaux suspects et accusés de sorcellerie, du fait de leur érudition en savoirs occultes. Les morceaux de rap véhiculent donc ici des cadres d'interprétation et de représentation du monde, réfléchissant des logiques de pensée propres à un contexte socioculturel particulier.

Toutefois, le cas de Roda N'No démontre que la pratique musicale ne se réduit pas à une expression de normes sociales et de représentations culturelles intériorisées, comme l'association entre vieillard et sorcier, mais de même que l'affirment les travaux réunis par Sara Fila-Bakabadio et Giulia Bonacci autour des musiques populaires, qu'elle entretient une « interpénétration avec la réalité sociale » (Bonacci, Fila-Bakabadio, 2003 : 9), en introduisant des dynamiques nouvelles et des instruments d'action sur ce réel. Dans le cas étudié, cette faculté d'action sur les ordres sociaux existants s'exerce et se donne à voir par un traitement de l'accusation de sorcellerie qui contraste nettement vis-à-vis des pratiques traditionnelles d'élucidation des causes du décès.

Au Gabon comme dans d'autres pays d'Afrique, une mort n'est jamais naturelle, et le décès d'un proche fait souvent l'objet de discussions et de réunions dans les familles pour déterminer les causes de la mort, ou le coupable du crime (Thomas, 1982). Ces réunions, étapes importantes du rite funéraire, se tiennent sous la direction des aînés sociaux qui orientent la discussion et l'élucidation des causes de la mort. Réglé selon une idéologie gérontocratique, le rite funéraire est un cérémoniel « codifié par ce référentiel capital que constitue le mythe et contrôlé par les ancêtres dont les patriarches sont ici-bas les représentants » (Thomas, 1982 : 11). Selon les ethnies, ce rituel peut prendre diverses formes; ainsi chez les Fang Nzaman, la mort s'accompagne souvent de danses d'initiés, ainsi que d'échanges et de discussions entre les différentes parties de la famille du défunt (paternelle et maternelle). Les enfants, bien qu'ils soient présents et parfois pris à parti, ne sont pas habilités à intervenir librement, et le rôle de porte-parole des familles est exercé par les aînés. L'accusation ciblée fait ainsi l'objet de règles et de codifications échappant bien souvent aux moyens d'action et de prise de parole des jeunes.

manifestation du bouleversement des imaginaires et des réseaux sociaux » (De Boeck, 2000 : 32).

${ }^{33}$ Les rappeurs reproduisent ce système hiérarchique lié à l'aînesse : se répartissant par génération, ils s'accordent davantage de respect en fonction de leur ancienneté dans le mouvement. Des observations similaires sur la distinction entre des " grands » et des " petits » et des privilèges différentiels en découlant sont relevées par Bob White auprès des groupes de rumba de Kinshasa (White, 2008). 
$\mathrm{Au}$ travers de ce type de texte, Roda N'No formule donc une accusation rompant avec la relégation des jeunes en dehors des prises de décision, et exprime une résistance des cadets sociaux vis-à-vis des ordres de contrôle du pouvoir subis face à la mort. Dans d'autres pays d'Afrique Centrale, des phénomènes proches de soulèvement des cadets contre la mainmise des ainés sur l'organisation sociale sont relevés. Au Congo, Jean-Aimé Dibakana (2005) note qu'en contraste avec les rituels ordaliques autrefois pratiqués par les aînés pour sanctionner les sorciers, « aujourd'hui, se passant des procédures et valeurs familiales, les cadets s'en prennent physiquement aux aînés accusés de sorcellerie »(Dibakana, 2005 : 146). Cet auteur analyse les réactions de plus en plus violentes à l'encontre des aînés accusés de sorcellerie comme une " réinvention des pouvoirs des aînés par les cadets sociaux » (ibid.), possédant des effets sur l'organisation et sur le fonctionnement social. Dans une autre étude d'anthropologie urbaine réalisée à Kinshasa, Ivan Vangu Ngimbi (1997) analyse pour sa part les conduites tenues par les jeunes autour de la mort durant les années 1990 comme une forme de protestation sociale. Prenant en charge les animations funéraires à la place des aînés sociaux, les jeunes s'approprient les rites de deuil ; par un ensemble de conduites perçues parfois comme des "obscénités ", ils inventent une "théâtralisation "new-look" du vécu mortuaire ", en vue de répondre à un double besoin : "un procès des rapports sociaux et un mode populaire d'action politique » (Vangu Ngimbi, 1997 : 17). Les pratiques musicales des rappeurs et les conduites des jeunes kinois se rejoignent en ce qu'elles mettent en place un procès des rapports de domination des aînés sur les cadets. De même que les réinventions des rites funéraires ou les actes de violence à l'égard des anciens, la pratique musicale rap charrie de nouvelles conduites face à la mort et face à la question de la sorcellerie, et constitue une forme $d^{\prime}$ action sur l'ordre social et symbolique en présence.

Toutefois à nouveau, cette posture singulière vis-à-vis des devoirs de respect aux ainés ne peut être comprise dans toute sa complexité sans prendre en considération l'importance que joue l'expérience initiatique dans le rap de Roda N'No. Rappelons d'abord que les rites de passage gabonais, souvent traversés en réponse à un sentiment de blocage "mystique » et d'attaque sorcière, sont aussi structurés autour du rapport aîné / cadet qui possède une importance notoire dans la vie sociale gabonaise. Jean-Ferdinand Mbah, dans sa description du rituel de posthectomie (ou circoncision) chez l'ethnie nzébi du Gabon rend compte de l'importance de cette structure initiatique dans la production de hiérarchies sociales entre aînés et cadets et dans la gestion des conflits qu'ils peuvent générer (Mbah, 1997: 50-54). Du côté de l'ethnie mitsogho, Julien Bonhomme relève que parmi les catégories hiérarchiques organisant la société secrète du bwiti (ainés/cadets, homme/femme, initié/profane), " la relation entre aînés et cadets est prise dans une relation d'ordre supérieure entre vivants et ancêtres »(Bonhomme, 2006 : 1947). La rencontre du néophyte avec ses esprits ancêtres, au sommet de la hiérarchie 
initiatique, et son accession au statut d'initié conduisent à une redéfinition de la place dans l'échelle générationnelle, ou du moins à la construction de zones plus labiles entre les catégories sociales.

Pour le cas de Roda N'No, ces remarques sur l'importance de l'initiation dans la manière de redéfinir ou de défier des frontières socialement instituées - comme celles distinguant les catégories d'aînés et de cadets -, se répercutent dans l'activité musicale, où elles sont subtilement réinvesties comme outil de contestation. Roda N'No explique ainsi les raisons de la transgression qu'il s'autorise dans ces morceaux :

«Par rapport à ce que tu disais, je peux expliquer ça aussi par le fait que je ne suis pas jeune en esprit ; je peux être moi aujourd'hui qui parle, mais c'est pas moi. Souvent je parle au nom de N'no Missoune, N'no là vient de N'no Missoune, qui a vécu bien avant moi. Je ne l'ai pas connu. Mais en m'initiant, moi je l'ai vu. C'est lui qui est vieux, mais c'est peut-être lui qui parle à travers moi. Ça peut être aussi toujours les vieux qui parlent à travers moi [silence].

Parce que Roda N'no est vieux. [...] Peut-être pour dire que j'ai beau être le benjamin, je suis le chef dans l'autre monde. Et quand je reviens, mon kombo, c'est Nkorebore. Un peu le sauveur, comme un Jésus ». Roda N'No, 1/8/2011, Libreville.

Durant son initiation, Roda $\mathrm{N}^{\prime} \mathrm{No}$ est entré en contact avec un esprit ancêtre, N'No Missoune, et s'est découvert un autre soi : "mystiquement vieux ", "fatigué », "se trainant avec un bâton », ce personnage était incarné par le nom « Nkorebore » et assumait un rôle de sauveur. Dans les propos cidessus, Roda N'No justifie ses accusations subversives à l'égard des aînés par le recours à une expérience initiatique dont l'un des principes tient dans la transformation des rapports entre aînés et cadets. La rencontre avec les esprits-ancêtres et les modifications de son statut dans la hiérarchie des aînés et des cadets est convoquée dans son activité musicale. Elle y résonne en partie avec un discours égotrip et des techniques de valorisation de soi basées sur l'identification à l'esprit Nkorebore et l'invocation de l'ancêtre, celles-ci légitimant du même coup les prises de position adoptées et la transgression des codes régissant les rapports entre ainés et cadets.

En outre, c'est en se présentant par sa musique comme un défenseur des savoirs, des modes de vie et des messages des anciens du village (qui expriment une certaine fierté face à sa recherche dans les profondeurs de la langue fang), que Roda N'No parvient à légitimer une posture qui déjoue les frontières entre aînés et cadets. Tout en insufflant des nouvelles dynamiques à l'ordre des rapports sociaux par sa pratique d'un genre musical inspiré de l'étranger, Roda N'No inscrit les dénonciations auxquelles il procède dans la continuité des transformations induites par l'expérience initiatique et dans le respect des codes religieux permettant de contourner les frontières sociales.

Pour ajouter un élément concernant cette combinaison entre respect de codes implicites et résistances contre les ordres de domination dans la 
pratique musicale, on notera que dans ses accusations, Roda $N^{\prime}$ No ne s'en prend qu'à des parents de son lignage maternel, et ne se réfère à aucun moment à ses parents paternels. Cette dénonciation élusive, omettant certains acteurs de son vécu familial et certains éléments découverts lors de son initiation, s'enracine dans une règle du système de parenté fang. Chez cette ethnie, l'enfant bénéficie en effet d'un traitement plus clément auprès des membres de sa parenté maternelle, tandis qu'il est sujet à davantage d'interdits et de devoirs du côté paternel. Cette règle résulte d'une distinction établie entre deux types de relations familiales ayant cours au Gabon : celle entre « les parents que l'on respecte, et les parents que l'on plaisante » (Mayer, 1992 : 157). Comme le note Raymond Mayer, chez les Fang patrilinéaires, la branche maternelle est le «refuge absolu pour un individu» (ibid.), ce pourquoi Roda N'No se permet de procéder à des accusations dans sa parenté maternelle qu'il ne peut formuler en direction de son lignage paternel.

Par un agencement complexe de ruptures et de continuités vis-à-vis des ordres sociaux en présence, la condamnation sorcière observée chez Roda $\mathrm{N}^{\prime} \mathrm{No}$ se construit donc dans une intersection entre les règles de l'exercice rap, le procès des ainés sociaux et la reconfiguration du vécu individuel induite par l'expérience initiatique. Il réinvestit de manière subtile les acquis de l'initiation pour procéder à des actions de résistance à la domination gérontocratique et contourner les ordres sociaux, élaborant dans sa musique un lieu de dissolution de certaines frontières. Dans la continuité des œuvres séminales de Fredrik Barth sur l'ethnicité (1969) ou de celles de Michèle Lamont sur les frontières sociales et identitaires (Lamont, 1992 ; Lamont \& Volnar, 2002), ces remarques mettent en évidence les modes de construction sociale des frontières, et leur dimension éminemment perméable, soumise aux stratégies des acteurs sociaux.

Nous venons de voir pour le cas des rapports hiérarchiques entre aînés et cadets comment la pratique musicale reconfigure des frontières socialement construites entre différentes catégories, en s'appuyant sur les matériaux symboliques et religieux offerts par les sociétés initiatiques locales. Au demeurant, cette observation ne vaut pas que pour les limites entre les catégories sociales, et nous allons maintenant voir qu'elle joue aussi sur les frontières symboliques structurant le mode de conception du réel et de l'imaginaire sorcier.

\section{MONTRER L'INVISIBLE : UN MORCEAU DE RAP ENTRE DEUX MONDES}

Pour Roda N'no, la musique constitue un instrument de dénonciation des pratiques de sorcellerie des aînés et du contrôle du pouvoir qu'ils opèrent. De fait, son écriture musicale agit dans un champ de rapports de force et de pouvoir habituellement compris dans l'univers du «mystique » et de la nuit. S'exprimant à ce sujet, il explique de la manière suivante : 
"Le fait de dire ça comme ça ouvertement, pour moi, c'est pour que les gens sachent. Le "vampireux », après il va avoir peur de toi le jour, parce que tu as dit ça ouvertement. Comme ça, je les mets au défi aussi ». Roda N’no, 12/05/2011, Libreville.

Au Gabon, la notion de vampire désigne l'acte de sortie nocturne hors du corps par l'esprit, ainsi que l'organe de sorcellerie (habituellement localisé dans le ventre). Le "vampireux » représente quant à lui celui qui opère ces actions, en d'autres termes, le sorcier.

Pour mieux comprendre ces propos de Roda N'no et dans la continuité des éléments présentés précédemment concernant sont initiation, il faut savoir que chez une partie des groupes ethniques gabonais, et notamment chez les Fang, la société se conçoit de manière duale. Au monde diurne du visible et des vivants répond invariablement un "monde nocturne, invisible au commun des mortels et parallèle au monde du jour» (Mba Ndzeng, 2006: 36). Cet univers est réservé aux individus dont l'évu, ou l'organe de sorcellerie, a été éveillé, ceux que Roda N’No intitule les «vampireux ». Les sorciers mais aussi les initiés et personnes ayant activé cet organe se retrouvent dans le monde nocturne où ils s'affrontent et se voient, dans un espace invisible au commun des mortels. Voir l'autre signifie ici découvrir son double nocturne et l'observer dans le champ de bataille spirituel fréquenté nuitamment par les sorciers. Lors de son initiation, Roda N’No a pénétré cet univers nocturne et s'est ouvert à une nouvelle forme de vision: celle décryptant les actions dissimulées des sorciers, celle permettant d'appréhender les acteurs du domaine mystique.

Dans un article traitant des fondements cognitifs des représentations liées à la sorcellerie, Julien Bonhomme (2005b) reprend ces observations concernant la confrontation entre un monde visible et « un monde invisible auquel seuls certains ont accès » (ibid. : 2). Il y analyse que l'invisible ne désigne pas en tant que tel une destination ou un espace identifiable, mais bien plutôt une modalité d'action, un mouvement, un acte consistant à sortir de soi pour consommer la force de l'autre, et ce sans avoir été vu de ce dernier. Le cœur de la représentation sorcière tiendrait donc davantage sur la sortie en vampire et l'acte de fondre sur sa proie de façon dissimulée, que sur un espace symbolique identifiable. De fait, c'est en réalité la notion d'invisible et "l'absence de perception visuelle" au moment de la sortie en vampire qui formeraient « la racine des représentations liées à la sorcellerie » (ibid.).

En résonance avec ces dichotomies nocturne/diurne et visible/invisible qui organisent la société fang et les représentations de la sorcellerie, Roda N’No explique ainsi les modifications apportées par l'initiation dans son comportement :

«Moi déjà, ça m'a ouvert mon esprit mystiquement. Aujourd'hui, si tu viens chez moi la nuit, je ne suis plus celui qui dormait paisiblement comme avant, qui ne 
voit rien le jour, qui ne voit rien la nuit. [...] Et moi aujourd'hui je pense pouvoir dire que si tu viens chez moi la nuit, je te vois.». Roda N’No, 1/8/2011, Libreville.

Ayant consommé le «bois sacré » (l'iboga) et accédé à l'univers nocturne, il a maintenant conscience des origines de son mal et de ses persécuteurs. Roda $\mathrm{N}^{\prime}$ No conçoit alors ses textes comme un moyen de faire savoir aux sorciers qu'il a connaissance de leurs forfaits, en d'autres termes qu'il les a vus lors de son initiation. Le morceau de rap opère une fonction singulière puisqu'en désignant de manière explicite les auteurs du méfait, il constitue une forme de contre-attaque par la mise en accusation qu'il exprime. Tandis que l'initié se transporte dans l'univers nocturne lors de son voyage initiatique, dans le but de voir ses attaquants et de les contre-attaquer, Roda N'No utilise pour sa part le rap comme un médiateur entre ces deux versants complémentaires de la société, le monde diurne et le monde nocturne, l'univers des humains et celui des sorciers. Il insiste d'ailleurs dans les propos cités à la page précédente sur cette intention de ramener dans l'univers du «jour» le combat entamé contre les hommes de la nuit et de les défier « ouvertement » par son rap. Bien qu'il fasse savoir au grand jour l'origine du mal, l'acte de dénonciation ou de contre-attaque se réalise dans un entre-deux associant visible et invisible : tout en déclinant son identité, Roda N'No peut atteindre sa proie sans être visible au moment de l'attaque, puisqu'il se dissimule derrière le support sonore du morceau de rap, de même que derrière un masque. Dans un interstice entre le visible du commun des mortels et l'invisible domaine des sorciers, le rap révèle au grand jour ce qui ne peut ordinairement être vu que la nuit.

Cette position interstitielle du morceau de rap, qui rompt avec les ordres coutumiers de déroulement des accusations, n'est pas sans provoquer des tensions et des inquiétudes dans l'entourage de Roda N’No. Lors de la parution du morceau accusatoire «Mayi», des proches de son village s'inquiétèrent de cette dénonciation ouverte, craignant que ses accusations n'entrainent les représailles des suspects désignés.

Toutefois, malgré ces appréhensions, quelques années après la parution de son album, Roda N'No constate qu'il a survécu aux bourreaux de ses parents, et qu'il est parvenu grâce à son morceau de rap à marquer une rupture vis-àvis du schéma de persécution sorcière, en procédant lui-même à une forme de contre-attaque et en reprenant le contrôle de son existence. En outre, bien qu'il procède à des accusations à caractère subversif, sa démarche de mise en liaison des espaces ruraux et urbains et sa mise en relief des savoirs recueillis chez les aînés de Makokou lui valent le respect et les encouragements des anciens du village, confirmant finalement la portée des productions musicales sur la construction d'espaces transversaux entre différentes catégories, ici celle distinguant aînés et cadets. 


\section{CONCLUSION}

Nous avons vu dans cet article comment l'investissement du genre rap par certains jeunes Gabonais, animés d'un désir de mise en avant de particularismes dans le marché musical, avait pu conduire à une rencontre et un entrelacement des logiques de l'univers rap à celles de l'univers sorcier. La description du parcours et de l'œuvre d'un de ces artistes, Roda N'No, a par exemple mis en exergue la coexistence de deux types de dialogue entre le rap et l'univers sorcier : cet artiste emploie en un sens le style égotrip pour défier ses détracteurs mystiques et magnifier son pouvoir sorcier, et convoque de l'autre l'univers du mystique et de la sorcellerie afin de particulariser son rap et de pénétrer les réseaux de la world-music.

Certes, Roda N'No se distingue sous plusieurs aspects de ses pairs rappeurs, et il ne constitue pas une figure d'exemplarité des pratiques ou attitudes ayant cours dans le milieu du rap à Libreville. Pourtant, son récit de vie n'en constitue pas moins un outil adéquat pour appréhender les dialogues entre la pratique musicale et l'impulsion de nouvelles dynamiques sociales et symboliques, en l'occurrence celles de l'imaginaire sorcier. En considérant que les individus sont producteurs du système social, et non réceptacles d'un ordre qui s'impose à eux (Bertaux, 1997), on ne peut manquer de relever l'intérêt de la méthode biographique et de l'attention portée à des "personnalités-carrefour» (Morin, 1980) pour appréhender les dynamiques du social et la porosité des catégories qui le composent. Le cas de Roda N'No m'a intéressée ici non pas car il constituerait un archétype du rappeur gabonais, mais parce qu'il témoigne de la manière dont un individu s'est emparé d'une forme musicale pour procéder à des modifications de son univers social, en connectant l'ensemble des normes et des contraintes propres aux différentes sphères au sein desquelles il évolue ou qu'il aspire à intégrer (la sphère familiale, le milieu hip-hop librevillois, le marché international de la musique).

Sur un plan épistémologique, ces observations confirment aussi la pertinence d'une étude des pratiques musicales pour appréhender la construction des modes de catégorisation de la vie sociale, territoriale ou symbolique. Le cas de Roda N'No témoigne d'une manière originale de se jouer des frontières sociales et symboliques: par une technique de triangulation, les référents initiatiques et la question du mystique sont convoqués dans la musique pour contester des hiérarchies gérontocratiques. Le morceau de musique configure un espace labile où se discutent et où se mettent en mouvement les ordres sociaux, pour voir émerger des passerelles entre les catégories de rural et d'urbain, d'ainés et de cadets, de visible et $\mathrm{d}$ 'invisible. Des études sur la ville ont mis en exergue la façon dont le maintien ou la transformation des frontières identitaires s'appuyaient sur « des éléments territoriaux, architecturaux et topographiques» (Hayot, 2002), d'autres sur les classes sociales ont souligné l'importance du gout dans la 
production de cultures de classe et de distinctions sociales (Bourdieu, 1979). L'analyse des œuvres d'un rappeur gabonais confirme que la musique s'inscrit aussi parmi ces domaines de redéfinition des frontières sociales, et l'importance d'une anthropologie des pratiques musicales pour comprendre le rôle de cette ressource symbolique «dans la création, le maintien, la contestation, ou même la dissolution de différences sociales institutionnalisées » (Lamont, Molnar, 2002 : 168).

\section{BIBLIOGRAPHIE}

Alfred ADLER, Roi sorcier, mère sorcière. Parenté, politique et sorcellerie en Afrique noire, Paris, Éditions du Félin, 2006.

Kali ARGYRIADIS, « Réseaux transnationaux d'artistes et relocalisation du répertoire "afro-cubain" dans le Veracruz ", Revue européenne des migrations internationales, vol. 25, n², 2009, [En ligne]. URL : http://remi.revues.org/index4955.html.

Michelle AUZANNEAU, «Le rap, expression de dynamiques urbaines plurilingues. Le cas de Libreville », Plurilinguismes, $n^{\circ} 18,2001$, pp.11-47.

Julien BARRET, Le rap, ou l'artisanat de la rime : stylistique de l'égotrip, Paris, L'Harmattan, 2008.

Fredrick BARTH, "Les groupes ethniques et leurs frontières", in Philippe POUTIGNAT et Jocelyne STREIFF-FENART, Théories de l'ethnicité, Paris, Presses Universitaires de France, 1995 (1969), pp. 203-249.

Florence BERNAULT, "Magie, sorcellerie et politique au Gabon et au CongoBrazzaville ", in Marc MVE BEKALE, Démocratie et mutations culturelles en Afrique noire, Paris, L'Harmattan, 2005, pp. 21-39.

Florence BERNAULT, Joseph TONDA, "Dynamiques de l'invisible en Afrique ", Politique africaine, vol. 79, 2000, pp. 5-16, [En ligne]. URL : http://www.politiqueafricaine.com/numeros/pdf/079005.pdf.

Daniel BERTAUX, Les récits de vie. Perspective ethnosociologique, Paris, Nathan, 1997.

Giulia BONACCI, Sara FILA-BAKABADIO (dir.), Musiques populaires: usages sociaux et sentiments d'appartenance, Paris, Centre d'Etudes africaines, 2003.

Julien BONHOMME, Le miroir et le crâne, parcours initiatique du bwete misoko (Gabon), Paris, CNRS Edition, 2005a.

Julien BONHOMME, «Voir par derrière. Sorcellerie, initiation et perception au Gabon », Social Anthropology, vol. 13, n³, 2005b, pp. 259-273 [En ligne]. URL: http://julienbonhomme.ethno.free.fr/Texts/ Voirparderriere.pdf

Julien BONHOMME, «La feuille sur la langue, pragmatique du secret initiatique », Cahiers Gabonais d'Anthropologie, n¹7, 2006, pp.1938-1953.

Pierre BOURDIEU, La distinction. Critique sociale du jugement, Paris, Éditions de Minuit, 1979. 
John CINNAMON, "Fan (Nzaman) », Revue Gabonaise des Sciences de l'Homme («Alphabet Scientifique des Langues du Gabon - Acte du séminaire des experts »), n², 1990, pp. 175-183.

Émilie DA LAGE, Des chants au monde, la production de l'authenticité musicale : réseaux, dispositifs et médiations, Coll. « Thèse à la carte », ANRT, 2000.

Émilie DA LAGE, 2009, «Politiques de l'authenticité », Copyright Volume, n6 (Géographie, musique et postcolonialisme, 2009, pp. 7-32.

Filip DE BOECK, «Le "deuxième monde" et les "enfants-sorciers" en République démocratique du Congo », Politique Africaine, n80, 2000, pp. 32-57.

Jean-Aimé DIBAKANA, «Décès d'enfant, désordre familial et mésaventures de l'oncle : la ré-invention des pouvoirs des aînés sociaux dans le Congo contemporain », Anthropologie et Sociétés, vol. 29, n¹, p.145-165, 2005.

Jean-Pierre DOZON, «Les métamorphoses urbaines d'un "double" villageois », Cahiers d'études africaines, Vol. 21, n81-83, 1981, pp.389-403 [En ligne]. URL : http://www.persee.fr/web/revues/home/prescript/article/cea_0008-

0055_1981_num_21_81_2321

James W. FERNANDEZ, Bwiti. An ethnography of the religious imagination in Africa, Princeton, Princeton University Press, 1982.

Samuel GALLEY, Dictionnaire Fang-Français et Français-Fang, suivi d'une grammaire fang, Neuchâtel, éditions Henri Messeiller, 1964.

Peter GESCHIERE, Sorcellerie et politique en Afrique. La viande des autres, Paris, Karthala, 1995.

Peter GESCHIERE, "Sorcellerie et modernité : retour sur une étrange complicité », Politique africaine, $\mathrm{n}^{\circ} 79,2000$, pp.17-32.

Karim HAMMOU, «Artistes, professionnels, stars. L'histoire du rap en français au prisme d'une analyse de réseaux », in Béatrice JOYEUX-PRUNEL et Luc SIGALO SANTOS (Ed.), L'Art et la Mesure. Histoire de l'art et méthodes quantitatives, Paris, Editions rue d'Ulm, 2010 [En Ligne]. URL: http://halshs.archivesouvertes.fr/halshs-00471732/fr/

Ulf HANNERZ, Explorer la ville, Paris, Ed. de Minuit, 1983.

Alain HAYOT, «Pour une anthropologie de la ville et dans la ville : questions de méthodes ", Revue européenne des migrations internationales, vol. 18, n³, 2002 [En ligne]. URL : http://remi.revues.org/index2646.html.

Christine HENRY, Emmanuelle TALL, "La sorcellerie envers et contre tous », Cahiers d'études africaines, 1-2, n 189, 2008, p. 11-34.

Jean-Marie HOMBERT, "Présentation de l'Alphabet scientifique des langues du Gabon ", Revue gabonaise des Sciences de l'homme, n², 1990, pp. 105-112 [En ligne].

URL :

lyon.cnrs.fr/fulltext/gabon/revue2/Hombert_1990_ASG.pdf

http:/ / www.ddl.ish-

Robert JAULIN, La mort sara. L'ordre de la vie ou la pensée de la mort au Tchad, Paris, Plon, 1967.

Michèle LAMONT, Money, Morals, and Manners: The Culture of the French and American Upper-Middle Class, Chicago, Univ. Chicago Press, 1992. 
Michèle LAMONT et Virág MOLNÁR, “The Study of Boundaries in the Social Sciences", Annual Review of Sociology, Vol. 28, 2002, pp. 167-195 [En ligne]. URL: http://www.jstor.org/stable/3069239.

André MARY, La naissance à l'envers, Paris, l'Harmattan, 1983.

André MARY, " Parcours visionnaires et passeurs de frontières », Manuscrit auteur, publié dans Anthropologie et sociétés, 27, 1, pp.111-130, 2001 [En ligne]. URL: http:/ / halshs.archives-ouvertes.fr/halshs-00204939/fr/

Raymond MAYER, Histoire de la famille gabonaise, Libreville, Centre Culturel SaintExupéry / Sépia, 1992.

Ludovic MBA NDZENG, Para-société et pouvoir politique au Gabon, Thèse de doctorat en ethnologie (dir. Philippe Laburthe-Tolra), Université de Paris V, 2006.

Jean-Ferdinand MBAH, Spectacle et idéologie dans la posthectomie au Gabon, Libreville, CERGEP - Editions Udégiennes, 1997.

Birgit MEYER, Peter PELS (eds.), Magic and modernity : interfaces of revelation and concealment, Stanford, Stanford University Press, 2003.

Henrietta MOORE, Todd SANDERS (eds.), Magical Interpretations, Material Realities. Modernity, witchcraft and the occult in postcolonial Africa, London, Routledge, 2001.

Françoise MORIN, «Pratiques anthropologiques et histoire de vie », Cahiers internationaux de Sociologie, vol. LXIX, 1980, pp. 313-339.

Sophie MOULARD-KOUKA, "Sénégal Yewuleen! ". Analyse anthropologique du rap à Dakar : Liminarité, contestation et culture populaire, Thèse de doctorat en anthropologie (dir. Alain Ricard), Bordeaux 2, 2008.

Roda N'NO, Alice ATERIANUS-OWANGA, Akamayong-Nkemeyong. Recueil de textes en langue fang nzaman, à paraître en 2012.

Anthony PECQUEUX, La voix du rap, Paris, L'Harmattan, 2007.

Anthony PECQUEUX, Le rap, Paris, Le Cavalier Bleu, 2009.

Nicolas PUIG, «Bienvenue dans les camps! », La pensée de midi, n² 28, 2009, pp.166177.

Yves RAIBAUD, "Les musiques du monde à l'épreuve des études postcoloniales », Volume!, n6, vol. 1-2, 2009, pp.5-16.

Louis-Vincent THOMAS, La mort africaine, idéologie funéraire en Afrique noire, Paris, Payot, 1982.

Joseph TONDA, Le Souverain moderne. Le corps du pouvoir en Afrique centrale (Congo, Gabon), Paris, Karthala, 2005.

Ivan VANGU NGIMBI, Jeunesses, Funérailles et contestation socio-politique en Afrique, Paris, L'Harmattan, 1998.

Bob W. WHITE, « La quête du lupemba : rumeurs, réussite et malheur en République démocratique du Congo », Sociologie et Sociétés, vol XXXIX, n², 2007, pp.61-77.

Bob W. WHITE, Rumba Rules. The Politics of Dance Music in Mobutu's Zaire, Durham NC, Duke University Press, 2008.

Patrice YENGO, «Le monde à l'envers. Enfance et kindoki ou les ruses de la raison sorcière dans le bassin du Congo ", Cahiers d'études africaines, Vol. 1-2, n 189, 2008, pp.297-323. 
Léa ZAME AVEZO'O, "La néo-oralité au Gabon: analyse de la figure du serpent dans les légendes urbaines ", in Ursula BAUMGARDT et Françoise UGOCHUKWU (ss. la dir.), Approches littéraires de l'oralité africaine, Paris, Karthala, 2005, pp. 229-251.

\section{DISCOGRAPHIE}

241, 241, Amour, unité, Nofia Sound, 2008.

Lestat XXL, Bienvenue à LBV, Zorbam Produxions, 2007.

Roda N'No, Meni, Zorbam Produxions, 2008.

Roda N’No, Akamayong / Nkemeyong, autoproduction, 2012 (à paraître).

V2A4, 1990, African Revolution.

RÉSUMÉ : A partir d'une ethnographie des rappeurs de Libreville, cet article analyse certaines répercussions de l'investissement du genre rap par les jeunes Gabonais, en interrogeant plus particulièrement les dynamiques sociales nées de sa rencontre avec les pratiques et représentations locales liées à la sorcellerie et au domaine qualifié populairement de "mystique ». Une description sommaire des thématiques de textes de rap et des relations ayant cours dans ce milieu musical présente d'abord les modalités de la pratique musicale dans ce terrain dit " miné » par la sorcellerie. Puis, l'analyse de l'œuvre et du récit de vie de Roda N'No, rappeur de l'ethnie fang, met en évidence les dynamismes insufflés par la rhétorique rap aux imaginaires sorciers et à leurs logiques sociales, cela en résonance avec les influences de l'expérience religieuse initiatique. Cet article s'intéresse finalement à la manière dont le rap est employé comme instrument de liaison ou de redéfinition des frontières entre différentes catégories de la vie sociale et symbolique : entre la province et la capitale, entre les aînés et les cadets sociaux, entre l'univers du jour (ou des humains) et celui de la nuit (ou des sorciers).

MOTS-CLÉS : Rap, Gabon, sorcellerie, initiation, frontière sociale. 